Voix et Images

\title{
Paul Chamberland la poésie, le vécu : recherche et expérimentation
}

\section{Caroline Bayard et Jack David}

Volume 2, numéro 2, décembre 1976

Paul Chamberland

URI : https://id.erudit.org/iderudit/200051ar

DOI : https://doi.org/10.7202/200051ar

Aller au sommaire du numéro

Éditeur(s)

Les Presses de l'Université du Québec

\section{ISSN}

0318-9201 (imprimé)

1705-933X (numérique)

Découvrir la revue

\section{Citer ce document}

Bayard, C. \& David, J. (1976). Paul Chamberland la poésie, le vécu : recherche et expérimentation. Voix et Images, 2(2), 155-172. https://doi.org/10.7202/200051ar d'utilisation que vous pouvez consulter en ligne. 


\section{Paul Chamberland la poésie, le vécu: recherche et expérimentation}

C. B. Ton cheminement depuis 1962 est non seulement intéressant mais aussi plein de rebondissements. Tu débutes avec Genèses, texte mythologique - harmonique (poui utiliser la formule de Philippe Haeck), tu continues sur ta lancée avec Terre Québec et L'Afficheur hurle (textes historiques - catastrophiques, toujours selon Haeck). Quelle direction prends-tu à partir de Éclats de la pierre noire d'où rejaillit ma vie et Demain les dieux naitront?

P. C. Éclats de la pierre noire, ce sont des poèmes de 1969-1970. Ça me paraît toujours être une sorte de moment de transition entre ce qui est avant et ce qui va suivre. Parce que déjà, dans ces textes-là (je l'explique à la fin du livre), c'est comme la fin d'un cycle, un cycle après lequel j'arrête d'écrire. Mais c'est très difficile à définir exactement: les ruptures, les re-départs, ce qui va suivre, dans quelle direction? (petit rire) Je ne sais pas.

Ce qui a précédé Éclats me paraît comme un préambule. Un moment précédant ce qui est manifesté dans Demain les dieux naîtront. Comme si la préparation était un pronostic. Mais ce point de vue n'est pas nécessairement objectif. Quant à la teneur, à ce qu'il y a d'existant, en soi, dans le livre, il me semble qu'on pourrait peut-être utiliser une image pour comparer mes deux phases. Jusqu'à Éclats, c'est comme si le Québec était le champ où mon imagination et ma pensée s'exerçaient. Avec la terre du Tiers-Monde, le paysage de la décolonisation, avec tous les mouvements, toutes les luttes des années 60 . Après deux ans de séjour en France, commence cette période de ma vie où j'ai écrit Éclats. Alors se prépare, s'accomplit ce silence, cette obscurité.

Avant c'était le Québec. Aujourd'hui c'est la Terre. Avant c'était Terre Québec, maintenant c'est Kébèk-Terre. Comme si le mouvement qui auparavant allait de la Terre vers le Québec allait maintenant du Québec vers la Terre. Tout le mouvement qu'on a appelé "contre-culture" ou "nouvelle culture", - pour moi, ce sont des étiquettes évidemment - 
constitue le champ ouvert de toutes ces transformations, un mouvement auquel je me sens participer profondément.

C. B. Dans le tableau d'ensemble de. la littérature québécoise contemporaine, entre les valeurs sérieuses et sûres des années 60 (Miron, Lalonde, Paul-Marie Lapointe) et les expérimentateurs, les révolutionnaires de la syntaxe (Brossard/Beaulieu) et les visionnaires du son (Duguay), où te situes-tu?

P. C. Je me sens proche de Raoul depuis longtemps. J'ai vu son propre développement. C'est une évidence qu'entre nos manifestations existent des différences énormes. Mais il y a une communauté d'inspiration, de pensée entre lui et moi. Sauf peut-être l'aspect "show", spectacle. Par nature je suis beaucoup moins spectaculaire que Duguay. Même s'il y a des ressemblances de goût et de mœurs entre nous depuis la fin des années 60 . C'est depuis le début des années 70 (on pourrait d'ailleurs choisir la "Nuit de la poésie "comme point de départ) que la poésie devient poésie “grand spectacle», non seulement lue, mais dite, parlée, chantée.

C. B. Tu t'apparentes à ces tentatives?

P. C. J'y ai participé, à ma façon, parce qu'on a eu beaucoup d'autres "nuits de la poésie », après la première, beaucoup de "shows" de poésie, auxquels j'ai participé assez régulièrement. Dans des collèges, des cafés, des boîtes. J'aime assez le faire et puisque j'ai le goût de le faire je comprends toujours, d'une fois à l'autre, à quelle nécessité profonde cela correspond. C'est par cela qu'une certaine identification, qu'une présence, celle de la poésie, de la vie, du processus même de la vie, peut être réanimée dans une lecture, un «show». Cela permet un contact direct avec les autres, avec des gens qui écoutent. Tu sens un feedback s'établir. Cela t'alimente, te permet de rentrer en contact avec la source. On s'imagine que c'est seulement dans une sorte de grand silence, de solitude qu'on va trouver la source. Mais on la trouve aussi dans une situation de communion. C'est également vrai.

C. B. Dans Éclats ce qui frappe le lecteur non averti mais sensible tout de même à la visualité d'un texte, c'est la typographie. Tout à coup tu te mets à utiliser des espaces blancs, des vides, de l'italique, des capitales, tu brises les mots, scinde le mètre. Quelles ont été les étapes de cette typographie expressionniste? Qu'est ce qui s'est passé avant Éclats et de quel besoin est né ce changement?

P. C. Ce qui me frappe quand je pense à cette période-là, c'est le goût de plus en plus prononcé de la matérialité du texte. Un goût qui va se reporter presque totalement sur la calligraphie. Le livre devient un objet et je veux prendre contrôle de tout ce qui constitue le médium, enrichir, multiplier les ressources du message. Tout cela s'est fait spontanément, est né d'un besoin spontané. C'est un travail qui s'est déroulé de lui- 
même, pour produire une unité entre la matérialité du texte et tous les niveaux de la signification.

C. B. Y-a-t-il eu des étapes qui ont précédé Éclats?

P. C. Dans l'Inavouable, qui est une œuvre simple et "classique" il y a tout de même des éléments qui annoncent Éclats mais ils sont très minces.

C. B. Dans une section d'Éclats, sous-titrée "la Pyramide de Chéops", tu adoptes une approche stylistique comparable à celle de Bissett: celle du collage - montage de textes dont l'origine est très différente (Rimbaud/Spinoza/René Vailland/des extraits de ton journal). Quel effet cherchais-tu à produire sur le lecteur?

P. C. Un effet qui était produit sur moi et qui s'est développé ensuite. II y a ce que j'appelle le "grand texte", fait de tous les textes, de tout ce que l'on peut lire. J'ai voulu établir un réseau métaphorique entre des textes qui peuvent être divers pour les faire se répondre les uns aux autres et finalement, si on observe le processus, on se rend compte que quand on pense, ou que l'on écrit, ce grand texte-là agit toujours dans notre texte. Alors je me dis: c'est assez naturel, logique, cohérent de reproduire le processus dans notre écriture.

J'ai été fasciné par le livre de Norman O. Brown (Body's love) qui est fait d'une quantité incroyable de citations qu'il découpe littéralement et intègre dans le texte. C'est un des exemples les plus évidents de ce processus-là et c'est dans un ouvrage où ce genre est habituellement peu utilisé. On attendrait plus cela en poésie, par exemple.

C. B. Tu crois que cela déclenche chez le lecteur un processus lié à la réflexion ou aux sensations?

P. C. Tout dépend à quel niveau cela joue. Parce que si on utilise des découpages de journaux c'est peut-être plus au niveau des sensations, c'est moins conceptuel. Parfois cela peut se manifester au niveau de mêmes mots que l'on peut retrouver dans une citation. Le mot appartient à deux contextes différents, alors on obtient un jeu métaphorique. Pour moi l'élément qui entre en ligne de compte là-dedans c'est le déplacement constant des sens, le jeu des connotations. Un des effets que cela peut produire est une sorte de destruction de chaînes associatives - réflexes chez le lecteur, et en même temps la création d'un canevas où se régénère un nouveau sens.

C. B. Le collage est alors, en quelque sorte, une nouvelle métaphore, une métaphore révolutionnaire?

P. C. C'est une métaphore qui tient compte de la pression contextuelle provenant de l'accumulation des livres, des textes, des journaux. On est entouré d'une telle quantité de papier que cela produit ce qu'on appelle en informatique "du bruit». Les messages se brouillent. C'est la Tour de Babel. 
J'accumule beaucoup de coupures de journaux - j'imagine que je pourrais faire un livre avec cela, à la fois texte et manchette, avec aussi des illustrations pour reproduire, manifester cette Tour de Babel et aller chercher aussi, par une sorte d'effet de dépaysement, des jeux de significations.

Si je prenais des coupures de journaux de 1969, 1970, elles produiraient un effet de dépaysement, surtout si je les plaçais en contexte avec d'autres, elles prendraient une valeur révélatoire par rapport à ces attitudes-réflexes que l'on a quand on lit des journaux. De là on pourrait faire une critique - pas une critique théorique, pas un discours sur mais une critique immanente au texte. Ce que j'ai fait une fois c'est de prendre une description des tortures que l'on avait fait subir à un prêtre anglo-chilien, sous le règne de Pinochet et puis j'ai monté cela avec une description que l'on trouve chez les alchimistes de l'œuvre au noir. Cela donne un sens totalement nouveau à un fait d'actualité par sa juxtaposition à un texte alchimique. L'effet voulu dans cette opération c'est une contagion. Le corps torturé du $X X^{e}$ siècle est introduit comme une immense œuvre au noir autour de la terre.

Le fait des textes dans l'histoire, au lieu de tout recréer soi-même, cela peut avoir quelque chose de plus puissant, cela renvoie le lecteur au grand texte qui est autour de lui.

C. B. Est-ce que les profonds bouleversements de ton texte, d'Éclats, ont été précédés, ou préparés, ou suscités même inconsciemment par certaines lectures?

P. C. André Breton oui, et aussi des lectures très suivies en linguistique et en sémiologie, cela a eu des répercussions sur les relations que j'avais avec la forme du texte. «Tel Quel» et Derrida ont aussi compté pour moi.

C. B. Ont-ils changé la vision que tu avais de ton propre texte?

P. C. Oui et aussi le processus de la production textuelle. A ce moment-là je lisais peu de littérature proprement dite, romans ou poèmes. J'étais davantage pris par une perspective philosophique, Althusser et Freud (en particulier j'ai lu son Interprétation des rêves) ou linguistique et critique avec Derrida. C'est vraiment ce qui a été déterminant. J'ai même pensé étudier avec Chomsky! La démarche scientifique d'Althusser, appliquée aux sciences humaines, cela m'a séduit. Foucault aussi, et toute cette question de l'épistémologie des sciences humaines.

C. B. Comment ces linguistes et sémioticiens ont-ils modifié le sens de ta production?

P. C. Ils ont précisé ma vision matérialiste du texte. Du texte qui se fait. Ils m'ont donné le sens de l'arbitraire du fonctionnement textuel.

C. B. L'utilisation de caractères d'imprimerie verts à la fin d'Éclats m'a intéressée. As-tu encore envie d'expérimenter avec des couleurs? 
P. C. J'en aurais bien le goût mais le problème c'est que c'est assez coûteux. Ce sont les conditions de production qui limitent les expérimentations. Mais je ne suis pas aussi attiré par les couleurs que par les dessins, la mise en page en fin de compte.

C. B. Je te cite: "la parole s'étranglait dans le murmure ou de raukes bégaiements ". Je me demande ce que cela révèle au sujet de tes rapports avec un système de référence aux fermes assises sémantiques et orthophoniques? Comment te situes-tu par rapport aux exigences du lecteur traditionnel?

P. C. Je ne poserais pas le problème de cette façon-là. Cela revient à cette histoire de la Tour de Babel, pour moi. Georges Bataille a dit quelque chose d'intéressant là-dessus lorsqu'il a essayé d'expliquer sa méthode, il a dit qu'il faisait du langage un usage classique, ce qui est relativement vrai. Sa prose est la prose classique française.

D'un côté, je cherche à écrire le plus simplement du monde; quand une pensée se formule je cherche à la transmettre telle qu'elle se formule sans modification qui décollerait du mouvement lui-même.

Sans rattacher ce processus à tout ce que Breton a défini comme l'écriture automatique, il me semble que ce qu'il reste de ces théories sur l'écriture automatique (sur cette question voir le texte de Breton dans "Point du jour") ce sont les limites et même l'impossibilité de l'écriture automatique en fin de compte. Je lisais aussi chez lui: "on a fait des plagiats d'écriture automatique». Mais comment peut-on plagier l'écriture automatique? Comment peut-on faire de la fausse écriture automatique qui ressemble à la vraie?

Il fait remarquer, à un moment donné, que quand on se lance dans l'écriture automatique il y a un second filet de texte qui intervient, qu'est-ce qui arrive? II y a les images qu'ont engendrées les mots qui viennent d'être dits et qui ont suscité de nouvelles images. Je cherche à saisir la pensée, le sentiment de l'image, tels qu'ils se font, sans jamais les forcer.

C. B. Tu cites Hermès: «Égypte, Égypte, il ne restera de toi que fables incroyables et rien ne durera de toi que des mots taillés dans la pierre." As-tu parfois l'impression qu'il en sera de même avec les textes écrits en ce moment au Québec?

P.C. Oui, non seulement au Québec, mais aussi sur la terre, it n'en restera rien. J'ai le pressentiment profond que tout cela passera au feu. C'est une vision apocalyptique, pas une prédiction, un pressentiment.

On pourrait aussi répondre d'une autre façon. Malgré la disparition matérielle de tous ces textes, il restera les monuments de papier euxmêmes, mais qui seront conservés comme ré-activables, pour le goût et le besoin des hommes futurs. Peu de chose. J'ai du mal à imaginer dans l'avenir des armées de spécialistes, tels qu'on les connait aujourd'hui 
fouillant tel secteur de la littérature de la fin du $X X^{e}$ siècle. J'ai beaucoup de mal à imaginer cela.

Peut-être restera-t-il la millième partie de ce qui s'est écrit et peut-être ce millième sera-t-il refondu dans une sorte d'œuvre, comparable à celle d'Homère. Laquelle est quand même le résultat d'une fusion de traditions orales de la société achéenne. Ce sont des images comme celles-là qui me viennent. Mon sentiment est aussi que nos langues actuelles, non seulement le français mais l'anglais aussi, n'ont pas beaucoup de temps à vivre. Il va y avoir un bouleversement linguistique et elles deviendront des langues mortes.

Je pense que peut-être même le langage, l'écrit auront un autre statut, beaucoup plus subordonné, beaucoup plus secondaire que maintenant. Du point de vue de la civilisation, ce qui me fascine c'est que l'écriture, par exemple à ses débuts, était un moyen de communication sacré, utilisé dans un contexte d'initiation, comme véhicule entre initiés. L'écriture a été à la base du pouvoir, du pouvoir royal, pharaonique, la masse étant analphabète.

Aujourd'hui tout le monde est alphabétisé. Cela nous fait beaucoup illusion. Nous sommes aussi analphabètes que les masses l'étaient à l'époque pharaonique, car ce qui compte c'est la communication électronique. La maîtrise des ordinateurs et du code électronique joue le rôle que l'écriture jouait dans le système pharaonique.

Est-ce qu'une conceptualisation électronique va sortir de l'avenir? Je n'en sais rien. Un autre phénomène de civilisation qui m'attire, qui est en train de se répandre autour de la terre, c'est le langage idéogrammatique, fondé, une fois de plus, de nouveau, sur le visuel et non plus sur le phonétisme. C'est un langage qui opère à un niveau élémentaire, comme le langage du code de la route, ou celui des modes d'emploi. Le langage idéogrammatique est en train de se développer et de se répandre. Comme celui du code Michelin - les sémioticiens ont étudié cela. C'est une langue silencieuse, une langue internationale qui est en train de se créer. Par l'idéogramme tous les peuples de la terre se comprendront même si leurs langages phonétiques sont différents. Quel essor cela prendra? Je n'en sais rien. Mais pourtant c'est déjà en progression. On pourrait déjà imaginer des livres faits à partir de cette idéographie. On pourrait faire des poèmes, dont le registre sémantique serait encore assez élémentaire, évidemment.

C. B. Les Brésiliens en ont déjà fait, les Japonais aussi.

P. C. Ils ne seraient pas dénués de poésie, de force poétique. La poésie peut très bien travailler dans l'élémentaire.

C. B. Mais il y a toujours un code, un système de référence sémantique qui précède ou finit le poème idéogrammatique. Tu n'échappes pas à une langue pré-existante. 
P. C. Si tu te sers uniquement de ces signes dont tout le monde connaît le sens, si tu t'en tiens uniquement aux signes connus (circulation, téléphone), tu évites le système de référence au code verbal.

C. B. Tu te définis comme un relai - générateur de la communauté utopique à venir. Quel effet cette définition a-t-elle eu sur ton écriture?

P. C. C'est une formule qui définit tout simplement le lieu de mon écriture. Comme si la substance de ce que j'écris était en grande partie dictée à partir du futur. J'entends ceci non dans un sens naîf, mais au sens de ce que le futur est réellement, dans le présent, en tant que tendances et possibilités. De sorte que l'on puisse développer des antennes qui permettent de capter dans le mouvement même du présent les potentialités qui pourront se développer dans le futur.

C'est en grande partie l'une des motivations profondes de mon écriture. Et aussi c'est ce qui maintient ma volonté critique de démolir les chaînes associatives, les «imprints » neurologiques qui nous empêchent de percevoir.

C'est dans ce contexte-là qu'un système mental comme le marxisme lui aussi ne peut pas échapper à ce travail de décapage, de dissolution. II y a tout un système mental, crée au $X \mid X^{e}$ siècle, qui bien évidemment en lui-même, et par son auteur, implique des antennes puissantes. Mais le texte marxiste, à travers tous les textes produits à partir de lui, s'est fossilisé. II faudra qu'il soit secoué, comme les autres, décapé. C'est la situation même de la théorie qui est en cause.

C. B. Tu conçois le marxisme comme une théorie, plus que comme la mise en pratique d'un mode d'emploi?

P. C. Oui, le marxisme est une théorie, au sens précis du terme, une théorie du monde récent. Ce qui n'est pas quelque chose qui se démolit aisément. II s'agit, d'ailleurs, d'en récupérer le noyau pour le porter plus loin. Ma démarche n'est pas une réfutation, elle n'a rien à voir avec une argumentation, elle se situe au niveau d'une transformation. Je ne suis pas anti-marxiste mais post-marxiste. Je me trouve dans une situation de post-marxisme. Ce qui ne veut pas dire que je parviens à l'être, de façon satisfaisante. Ça, c'est une autre paire de manches! Entre affirmer des intentions et pouvoir les réaliser, il y a toute une marge.

Quels sont les critères au nom desquels on pourrait évaluer un travail post-marxiste? II n'y a ni points de repère ni consensus mais des chercheurs isolés, qui ne peuvent même pas se communiquer leurs recherches de façon satisfaisante. II faut être résolument audacieux, pour rompre avec ce qu'on sent très bien comme entraves et rétrécissements. Une fois lancé dans ce mouvement-là il faut être extrêmement prudent.

C. B. Comme un explorateur en terre inconnue?

P. C. Oui, un explorateur est prudent à cause du terrain. Là c'est la même chose. C'est aussi vrai dans le domaine de la vie elle-même, dans 
nos relations, où on peut entrer en conflit avec l'ordre des valeurs, l'ordre légal, policier. II faut tenir compte de la répression possible. La prudence s'impose. Également au niveau du corps. II ne faut pas se détruire et gâcher le travail. C'est un peu comme la pratique de la schizophrénie ou de la paranoïa mais en les renversant, en choisissant d'en faire ce que j'appelle une métanoïa (Noïa = connaissance). La paranoïa est l'élément type de la situation moderne, urbaine, comme Burroughs l'a exploré en détail de façon rigoureuse et éclatante.

Mais en étant métanoïaque-schizophrène expérimental on peut être en même temps un auto-thérapeute, un militant du bonheur dans le domaine de l'expérience, en s'associant chaque fois que c'est possible à des compagnons chercheurs; les dangers de se faire enfermer en soi-même peuvent être évités.

C'est donc sur un fond d'audace, dans la confiance, que l'explorateur se lance, en assumant, avant de partir, la possibilité d'y rester. On ne peut pas jouer et tirer son épingle du jeu en même temps.

L'écriture pour moi, et c'est une des grandes différences par rapport au passé, l'écriture est elle-même une œuvre. Je prends l'expression classique: ce qui, d'une part n'enlève absolument rien à la rigueur, à la passion, mais implique que je ne peux écrire que si je vis, expérimente, agis dans un champ humain qui lui, est au centre.

Quelle que soit la différence de style, de forme, tous les textes que j'écris sont des rapports de laboratoire, et la chose est d'autant plus évidente que, si j'écris, mon mode de vie depuis sept ans, c'est le mode de vie communal. Aller choisir de vivre en commune, avec des enfants, dans un contexte d'expérimentation très grand, pour consacrer sa vie à l'écriture, on ne pourrait à première vue choisir de pire moyen. Ma vie n'est pas fondée sur l'écriture, comme priorité. Mais ce qui se passe c'est que le mouvement de l'écriture reste aussi fort mais il ne serait pas ce qu'il est, et les textes non plus ne seraient pas ce qu'ils sont, si je ne vivais pas ce que je vis qui a tellement l'air de m'empêcher d'écrire. C'est une contradiction mais une contradiction féconde.

J'écris par fragments. II y a toujours un côté plus ou moins inachevé dans mes textes. Un côté bâclé, rapide. Mais de toute façon pour moi la notation rapide est parfois le genre le plus rigoureux à utiliser. $\mathrm{Si}$ je regarde aussi dans la production anglophone, au hasard des revues (USA et Canada), je vois des similitudes de fonctionnement.

C. B. Jadis tu parlais de l'écriture comme d'une magie individuelle. Est-ce que la calligraphie, le collage, la typographie révolutionnaire ne t'aident pas à communiquer cette magie?

P. C. Ce que je me trouve à écrire vient de ce que je capte et, entre autres, de ce que les gens autour de moi vivent, sentent, éprouvent et je peux communiquer directement avec eux. Donc, cela se vérifie. Souvent les gens de la commune me disent aje n'ai pas besoin d'écrire, tu le 
fais pour moi ». Cela devient une sorte de concentration, dans l'écriture, de ce qui est vécu par les autres.

\section{B. Tu es scribe et voyeur en même temps?}

P. C. Scribe et témoin. Avec la double notion: celui qui peut donner lecture ou témoignage, et celui qui aborde et rend manifestes dans ses écrits ceux qui vivent. Je me vois comme un être d'antennes. Jusqu'à maintenant j'ai été encore trop prisonnier du rationalisme. J'espère que progressivement je vais fonctionner comme un intuitif. La raison est, je pense, clarté, mesure, possibilité, mais elle devrait être emportée par l'intuition.

Je parlais d'antennes, parce que je l'ai vérifié, en lisant mes poèmes, quand des lecteurs viennent me voir ensuite au café, au restaurant, sur la rue Saint-Denis : être une antenne c'est aussi capter ce que des gens vivent, ce que je ne connais pas, et le retransmettre. Je parlais du rapport de laboratoire: un individu peut dire ce qui lui est arrivé à lui. C'est vrai, il dit: je l'ai vérifié, éprouvé, vécu. Il faut qu'il y ait la marque individuelle, l'originalité du sentiment, de l'imagination, de la pensée. Je suis toujours fasciné par les gens avec qui je vis. C'est assez fréquent qu'on entende ce qu'ils disent et qu'on l'écrive, cela fait des poèmes extraordinaires ou un aphorisme extraordinaire.

C. B. Est-ce qu'avec la calligraphie tu essaies précisément de transmettre ce système de perception et de notation de voix différentes qui parlent à différents moments?

P. C. La calligraphie est le niveau d'équivalence de cette variété. La calligraphie est le dispositif qui permet de le faire. Comme une stéréo-écriture. Elle comporte cet élément de l'individualité: j'y ai été, je ne parle pas en l'air, je parle de ce que je sais. Comme l'explorateur qui dit j'y ai été. Mais de plus en plus de gens peuvent y aller!

Une calligraphie c'est finalement une graphie et jamais deux graphies ne seront semblables. C'est le «caractère d'imprimerie" qui arrive à être propre à chacun. La potentialité de ce moyen-là m'a toujours beaucoup motivé. Si tout le monde se mettait à calligraphier (non pas que je dise qu'on doive faire cela) chacun signerait ainsi son livre. J'ai un peu le sentiment que je pourrais m'abstenir de signer mes livres. La calligraphie est une signature en elle-même. II y aurait ce paradoxe de l'anonyme et de l'individuel qui surgirait. II y a une époque d'auteurs anonymes. Tout simplement: il faut être bien là quant au texte qu'on produit comme étant son texte. Être présent, l'authentifier en quelque sorte et ceci ne signifie absolument pas qu'il est votre propriété au sens où on dirait: "c'est moi qui ai pensé cela, c'est ma pensée ce n'est pas la tienne". Non, ce n'est pas du tout cela, c'est un lien commun qui n'est commun que parce que chaque individu l'enrichit, pas parce que chaque individu s'abolit, ce qui serait une fausse conception de l'auteur collectif. 
C. B. Tu parles aussi de l'exploration systématique du cerveau, de l'expérimentation du corps magique-astral. Est-ce que ta quête face à l'écriture et à la vie quotidienne s'apparente à celle de Péloquin ?

P. C. Je ne pourrai pas donner de réponse à cela. On s'apprécie. On est bons amis. J'ai fait un petit texte une fois sur Pélo, où j'expliquais comment je nous voyais dans le même astronef. On transcrivait, on transmettait d'une manière différente, mais à partir du même paquet d'ondes. Péloquin, au fond, est exactement un compagnon chercheur, comme Duguay, comme Denis Vanier, ou comme d'autres, inconnus.

C. B. As-tu essayé d'écrire des poèmes en collaboration avec d'autres écrivains?

P. C. Cela pourra se produire. Cela m'intéresse. C'est intéressant, cela n'empêche absolument pas la spécificité de chaque main, ce qu'on appelle le style.

C. B. Quand exactement, comment et pourquoi as-tu été amené à fonder la Fabrike d'Ékriture?

P. C. La Fabrike d'Ékriture est devenue par la suite le Laboratoire de poésie pratique. Cela a commencé avec mes deux années d'enseignement à l'Université de Montréal alors que je dirigeais un séminaire de création littéraire et où, bien sûr, il s'agissait d'expérimenter et non d'appliquer des recettes, d'écrire dans une perspective normative. Peutêtre y avait-il des fautes de syntaxe, de style. J'ai ensuite quitté l'université pour me joindre à un groupe de créateurs-animateurs réunis autour de Fernand Dansereau. Le Groupe s'est appelé In-Média. Dansereau était cinéaste, mais travaillait aussi avec le vidéo. II avait aussi l'habitude du bio-feedback, et de la télévision. L'équipe a réuni le théâtre, la peinture, la musique, la danse, la chanson, rassemblant des gens qui s'intéressaient à la dynamique de groupe, à l'animation.

Cela a consisté en un type de stage à la fois fondé sur la création artistique et sur un certain nombre de règles, les règles du jeu que l'on trouve dans la dynamique de groupe. On interroge le véhicule collectif, pas seulement celui de l'individu mais du groupe. C'est à ce moment-là que le nom de la Fabrike d'Ékriture m'est venu. J'avais déjà fait des ateliers d'écriture dans les écoles, avec des professeurs de français de l'enseignement secondaire qui voulaient faire face à leurs interrogations, à leurs angoisses à cause du nouveau programme-cadre de français.

Cela a été une expérience très intéressante. La créativité dans l'écriture est devenue un mode d'apprentissage. Apprentissage de soi-même, un mode de communication avec les autres, un apprentissage des ressources pédagogiques face à l'apprentissage de la langue. C'est ma commune qui m'a fait interrompre cette expérience-là parce qu'elle a absorbé mes énergies. 
C. B: A quel moment as-tu interrompu tes activités avec la Fabrike d'Ékriture?

P. C. Vers la fin de 1973. De nouveau, cela m'arrive d'y penser. J'ai envie d'en refaire.

\section{B. Avec des enfants ?}

P. C. Oui, j'aimerais beaucoup en faire avec des enfants, et des adultes, dans le contexte de l'enseignement. Mais je ne sais pas si les choses ont évolué pour que je trouve une situation plus favorable. Tout le cloisonnement du monde scolaire était étouffant (par exemple, les difficultés pour trouver un local). On était compartimenté du point de vue de l'horaire. Et puis aussi, tout retombait dans le vide, les gens se retrouvaient dans leur contexte habituel et tout le profit, s'il n'était pas complètement perdu, était bien rétréci. Cela a créé une sorte d'amertume. Je me suis dit à ce moment-là, n'est-ce pas peine perdue? C'est quand même attristant tout d'un coup de constater cela.

C. B. Les étudiants, les élèves étaient-ils très réceptifs à tes perceptions sur la matérialité du texte?

P. C. À l'université ou au niveau de l'école secondaire?

C. B. Aux deux niveaux.

P. C. Pas toujours. Certains oui. Pour moi c'était un mode de libération, de libération jusqu'au niveau musculaire, à cause des conditionnements de l'écriture jusque dans le corps.

Très souvent des gens se sont mis à prendre des feuilles énormes et à barbouiller, à écrire de toutes sortes de façons. Mais la plupart du temps ce qui se produisait c'est que les gens éprouvaient le besoin de se mettre à parler. C'était davantage sur le plan oral que graphique que les choses prenaient de l'ampleur. Cela m'a frappé. Mais j'ai rencontré mes limites: j'étais moins prêt que je ne pensais. Duguay était beaucoup plus apte à entraîner des gens dans une exploration de la musculature vocale.

C. B. De toute façon, il est nettement le poète de l'oralité.

P. C. Oui. Cela s'est passé assez souvent à la fin. Nos expériences se sont rapprochées de celles du centre Essalem en Californie qui fait des expériences avec le corps, la voix, les sens. On a fait des choses intéressantes, en improvisant beaucoup. Vers la fin, particulièrement, on s'est lancé dans l'exploitation des rêves. Je me souviendrai toujours d'un atelier de rêves que j'avais formé. C'était un groupe assez large, huit ou dix personnes. La plupart disaient: non, je ne veux pas raconter mes rêves. Je me suis dis: bon, il faut tâter le terrain. J'ai commencé à parler de certains rêves, les miens. A faire valoir la fécondité du rêve pour la transformation du soi, pour l'expression du soi. Puis les gens se sont mis à raconter tel rêve qu'ils jugeaient sans doute plus anodin. Et finalement ce qui m'a intéressé c'est que deux jours après ils racon- 
taient tous très facilement leurs rêves. C'est même allé jusqu'au point où on rêvait les uns des autres et je me suis rendu compte de la fécondité d'un tel atelier.

C. B. Tu mentionnes parfois les interdits que le poète doit affronter, dans l'intégralité de sa personne et de sa vie. Pourrais-tu en citer quelques uns?

P. C. Dans l'écriture, tous les interdits esthétiques. C'est un lieu commun maintenant de faire valoir la transgression. II y a des modes de transgression qui, du reste, tombent dans une recherche de la nouveauté pour la nouveauté. C'est curieux à dire mais il subsiste en général un interdit de la simplicité, d'abord parce qu'il y a de fausses simplicités qui finissent par renforcer les interdits. La vraie simplicité s'obtient comme un résultat au bout d'une ascèse ou d'une purification.

C. B. Et les interdits syntaxiques, orthographiques?

P. C. Oui, surtout les interdits orthographiques. Malgré le fait que l'orthographe du fait de la pression culturelle se transforme en débandade aujourd'hui. Pour dépasser la question de l'écriture, je puis penser à la démarche que Deuleuze et Guatarri définissent dans l'Anti-CEdipe. Pour moi, il y a une situation qui est "de nature" chez moi. Et c'est l'interdit autour de la sexualité. Cet interdit-là est métaphorique à bien des niveaux. Je pensais à Deuleuze et Guatarri, car l'interdit sur la sexualité, c'est un interdit vis-à-vis du corps comme moyen de communication. En ce qui me concerne je songe à l'interdit de la pédérastie. Pour moi c'est ce que j'ai à vivre, c'est un destin, je ne l'ai pas choisi. C'est un territoire schizo-sexuel, un territoire politique.

C. B. N'as-tu pas suivi une certaine trajectoire en ce territoire?

P. C. Certains livres ont compté pour moi, m'ont aidé à mûrir. Particulièrement celui de Toni Duvert, le Bon Sexe illustré. II parle de cet interdit pour démolir l'Encyclopédie du sexe (en six tomes publiée chez Hachette) qui, sous des allures libérales, n'est qu'un rafraîchissement de la sexualité bourgeoise, de la propriété privée, de l'autorité de la famille sur les enfants. Pour moi, je dirai que, de naissance, la sexualité est d'emblée un territoire où je me sens en transgression. Ou bien je me range, j'étouffe et je meurs. (On peut mourir et devenir un Monsieur très bien, dans ses livres, etc.) Mais ce n'est pas possible. Ce que je sens en moi comme force, mouvement, vitalité est au fond irrépressible. Cette voie-là je l'ai tentée d'ailleurs. J'ai passé à travers tout le modèle bourgeois et c'est en connaissance de cause que je le romps.

Cet interdit il faut le vivre dans la solitude, en essayant de rompre avec le ressentiment, l'amertume, l'agressivité, la rage que la société crée chez le déviant. Cela c'est le premier point. Parce que cela vous entraîne vers des modes de transgression qui sont aliénés, eux aussi. Vous devenez semblable à l'image monstrueuse qu'on plaque sur vous, l'image du child-molester. Duvert est très net là-dessus. II démystifie l'image en 
donnant des statistiques très précises - les enfants brutalisés ou assassinés par des "child-molesters", si on compare cela aux enfants maltraités ou violés par leurs propres parents c'est vraiment très disproportionné.

Ensuite, la structure paradoxale de l'interdit consiste en ce que l'image du monstre est chargée de masquer son contraire parce que ce qui est interdit, c'est la tendresse. Cela s'applique à d'autres univers et d'autres interdits mais particulièrement au monde homosexuel. Le grand interdit dans cet univers c'est la tendresse. Le cœur de l'interdit c'est ce qui serait la chose du monde à le moins interdire. Quel est ce paradoxe? C'est tous implement l'écrasement de la sexualité infantile. Parce qu'il s'agit de créer un bloc occidental chauviniste, mâle et bien connu. On est vissé dans son rôle et le rôle consiste à récupérer ce qui est récupérable dans l'homosexualité, le «butch" par exemple. Mais l'enfant, en partant, est complètement réfractaire à cela. C'est formidable tout ce qu'il faut pour dresser un enfant. L'interdiction d'une véritable communication érotique-amoureuse entre un adulte et un enfant: elle se passe de toute façon; toutes les mères aimantes sont pédérastes quand elles aiment leur propre enfant et c'est finalement ce qui rend un être affectueux et chaleureux, mais comme cela est protégé par le code familial les apparences sont sauves.

Certains contextes permissifs m'ont permis de vivre des choses que je n'avais même pas entrevues. Si l'enfant établit un rapport général avec l'adulte qui consiste dans ce qu'on appelle l'imitation (une appropriation en quelque sorte de ce que l'adulte vit) et que l'adulte réponde à cette demande dans une perspective non autoritaire de réciprocité et qu'on se rende compte que comme base de pédagogie c'est à la fois quelque chose de très simple et de très efficace, je ne vois pas pourquoi, lorsqu'on arrive au niveau de la sexualité, de la communication érotique, cela cesserait d'être vrai. Mais ce qui est très délicat, c'est comment opérer, avancer là-dedans. Il y a cependant des règles très simples qui suppriment des complexités inutiles. II faut se laisser guider par l'enfant. Ce qui est fascinant, à un moment donné, c'est comment un mode très nettement érotique pour moi, en transparence maintenant, de relations générales entre des enfants et des adultes, chatouiller par exemple, en soi cela fait partie des jeux érotiques; et cela a l'air de se dérouler très naturellement, mais en fait il y a là des bloquages, des nœuds. L'éventail d'une communication totale n'est pas établi.

Le fait de l'avoir vécu, dans une situation où ces "hang-ups "-là n'existaient plus, me l'a montré précisément. Souvent, c'est une autre forme subtile pour maintenir l'interdit, on réduit les choses, en disant: "oh oui c'est un goût particulier, la pédérastie ma foi, n'est qu'un goût particulier "... c'est subtil parce que la question n'est pas là, cela n'est ni faux ni vrai mais en disant cela on réduit la pédérastie à la singularité. Je dirai que si chacun est totalement lui-même et chaque relation totalement elle-même, comme je l'ai constaté maintes fois, c'est drôlement 
un "goût particulier" ce que peuvent alors vivre un homme et une femme entre eux! Cette situation-là, hétérosexuelle et monogame entre deux adultes, ce que l'on voit, c'est le modèle, on ne se rend pas compte de la richesse de «perversions" qu'un homme et une femme peuvent vivre dans leur relation. J'ai vu des êtres autour de moi (je pense à deux couples en particulier) qui vivent totalement leur relation et comme je la vis par symbiose avec eux, on se rend compte qu'ils inventent.

Dire que la pédérastie est un goût particulier, c'est ne rien dire du tout. Je pense que la pédérastie est un goût général. Je veux dire par là qu'une communication affective et érotique entre l'enfant et l'adulte me parait être une nécessité de l'espèce, celle de l'amour que l'espèce a pour ses petits. Si la communication avec l'enfant est totalement assumée, en termes érotiques, comme dans d'autres termes, à ce moment-là ce qui se passe c'est une sorte de re-fusion entre l'enfant et l'adulte, ce qui est mis en circulation et casse les moules bourgeois autoritaires, c'est que l'enfant ressuscite chez l'adulte et que l'adulte transformé par l'enfant prend une allure totalement nouvelle pour l'enfant. Chez ce dernier cela active un adulte. Cela correspond à ce que j'appelle l'hommeenfant en puissance chez l'un aussi bien que chez l'autre.

Un aspect fondamental de mon combat se situe là. Mon cuvre est làdedans, au moins autant que dans l'écriture ou dans le mode d'expérimentation de la vie communale. Je me sens un créateur aussi fortement dans l'érotisme que dans l'écriture. Ce qui est terrible c'est que jusqu'à maintenant, j'étais dans une situation paranoïaque telle que je ne pouvais pas me manifester. Cela commence à changer. Je peux me manifester auprès de plus en plus de gens qui sont amenés à vivre leur recherche sur le plan de leur propre sexualité, je me suis forgé le concept, un peu barbare, $d$ ' «isosexualité»: chacun vit sa singularité érotique, sa singularité d'être de désir et se retrouve semblable dans l'autre; alors il n'y a plus qu'une seule sexualité qui se manifeste d'autant de façons qu'il y a d'individus et de relations. Toutes les formes communiquent entre elles. Je pourrais renvoyer au texte de Fournier, le Nouveau Monde amoureux.

Ce qui est décisif, pour moi, là-dedans, c'est qu'il en va de ma propre vie. J'ai horreur de la clandestinité, de l'hypocrisie. Je me sens totalement étranger au monde de Jean Genet par exemple, et ce n'est pas là une condamnation mais une constatation. Ma trajectoire c'est d'ouvrir de plus en plus (et cela correspond au personnage du Prince de Sexamour) un territoire de créativité dans l'érotisme, en participation avec tous les autres. Si je n'y réussis pas, j'ai l'impression que mon équilibre va être compromis. Ce que j'éprouverais là ce serait comme un refus de ma nature elle-même. L'écriture ne m'apparaîtrait plus que comme une compensation.

C. B. Tu dis aussi: “je me sers de l'écriture en homme de science, je formule des hypothèses, je hasarde des théorèmes, j'enregistre des oracles, je traite de concepts, je fixe la trame de la conscience, je cherche 
à tirer les encéphalogrammes les plus précis de la performance linguistique de mon cerveau ".

Définirais-tu une certaine poésie actuelle et la poésie à venir, comme une œuvre de science?

P. C. Ce que je dirai maintenant, pour ajouter à cette formulation-là c'est que c'est une science telle qu'on peut l'entendre par le mot gnose. Un savoir qui implique une transformation de l'être. Le modèle scientifique, jusqu'à Einstein, c'était le modèle des sciences physiques fondé sur la séparation entre l'observateur et le champ observé. On a voulu faire la même chose en sciences humaines. C'est une belle rigolade. Et on peut voir dans quelle mesure Marx avait déjà subverti ce modèle-là. Le modèle scientifique, un savoir rigoureux, communicable, qui n'est pas de l'ordre des sentiments, un savoir opératoire c'est-à-dire qui permet des transformations, ne sera possible qu'en fusionnant les deux démarches, art et science. En remettant tout ensemble, en discernant les plans, mais aussi en les conjuguant. Sortir du logocentrisme, du rationalisme est important. L'écriture ne serait plus pratiquée comme une façon d'obtenir des bons textes et de perpétuer une sorte de consommation bourgeoise des beaux-arts, mais par tout le monde, à des degrés divers, comme un des modes de connaissance de soi-même.

On pourrait imaginer que chaque individu, pendant une phase de sa vie, pratiquerait l'écriture. Et ce que je veux dire par là c'est que chacun expérimenterait l'expression langagière, non pas nécessairement pour obtenir des résultats esthétiques, mais des produits qui le renseignent par feed back sur le processus en lui, sur son fonctionnement, dans son imagination et dans son langage. Certains passeraient vite parce qu'ils obtiendraient finalement des résultats aussi satisfaisants par d'autres façons. Certains pourraient arriver à garder cela comme un fonctionnement très profitable, et on pourrait à ce moment-là, les appeller des écrivains. Mais il serait entendu qu'être écrivain ce ne serait qu'une des façons d'être ce type de chercheur et de gnostique. Un rang aujourd'hui un peu mystérieux, privilégié, renversé souvent d'ailleurs par le mépris. Voilà ce que je vois au niveau de l'écriture. Cela se conjugue à l'exploration du rêve. Ce sont des choses qui sont déjà sues.

C. B. Tu as dit: «Du poème à l'acte poétique, tout est à réinventer. "Quel est ce tout?

P. C. Je pense que c'est réinventer la terre, l'espèce humaine. Mettre sur pied une écologie syntaxique. Une écologie de notre rapport à la nature, à nous-mêmes, tribale en somme. Le tenter par classe, par groupes du style "commune», ou dans un réseau de compagnons chercheurs. Selon les circonstances, les rencontres, les événements. C'est un tout qu'il s'agit de percevoir. Tout est dans tout. C'est la formule des gnostiques.

C. B. Celle de Raoul aussi! 
P. C. Oui, celle de Raoul aussi.

J. D. Les poètes américains de Black Mountain, Creeley, Olson, parlent souvent de leur lecture de l'univers. Lorsque tu parles d'encéphalogrammes, de tes perceptions de ce qui est au-dedans de toi, et sur la page, tu me sembles être leur frère. Les as-tu jamais lus?

P. C. J'ai constaté des similitudes. C'est assez naturel que cela se produise parce que il y a la mise en place en Amérique du Nord d'un nouveau mode de conscience, d'une nouvelle structure de l'esprit. Les Allemands ont une expression pour cela: Zeitgeist. $A$ travers le texte de Leary j'ai senti que je vivais ce qu'il vivait, comme beaucoup d'autres en Amérique et autour de la terre sans doute. J'étais branché à un centre qui nous activait, qui était réellement l'auteur. Quand j'ai décidé de faire cette photo (sur la couverture de Demain les dieux naîtront) c'était pour signifier: je suis un scribe, (c'est la posture des scribes égyptiens). Je ne suis pas auteur mais secrétaire. Le secrétaire doit avoir un bon instrument. Pouvoir écrire avec un maximum de richesse. Donc une appropriation de son matériau. L'art, au sens essentiel du terme, c'est l'art au sens que les constructeurs de cathédrales étaient des artistes finalement. Pas dans le sens d'une petite propriété bourgeoise, d'un territoire original, mais une appropriation individuée. Ce n'est pas le réalisme socialiste.

J.D. Lorsque tu te sens un véhicule, un instrument pour les autres et pour ce qui te traverse au moment même où tu écris, te sens-tu capable ou même en train de changer ce qui te pénètre?

P. C. Je pourrai dire que ce n'est jamais tranché en pratique. Cette source-là c'est le Soi. J'ai eu une vision déjà de cela. Mon ego ne doit pas disparaître. S'il n'y a plus d'ego, le soi ne pourra plus se manifester, il n'y aura plus d'instrument. Donc, ainsi que les mystiques islamiques ont développé cela, je suis le témoin. C'est-à-dire que Dieu se regarde et se fait être à travers moi. Pour reprendre leurs termes je suis Dieu, je renvoie un reflet à Dieu, je suis l'œil à travers lequel Dieu se regarde. A ce moment-là, ma densité propre doit être complètement développée. II n'y a pas de pertes. Et en même temps je suis l'une des radiations de ce soleil-là.

C'est une vision paradoxale, je l'ai eue un jour. Je voyais un soleil central, extraordinairement brillant et j'étais comme un mouvement de vibration à la périphérie de ce soleil-là, mais ce soleil-là n'était pas ailleurs qu'au centre de moi. De sorte qu'il était le centre pour tout le monde qui le percevait comme leur centre, il y avait une sorte de boucle. A ce moment-là, je me suis dit (et c'est la question de l'art et de la fidélité) : je ne dois pas être angoissé, je dois être le plus disponible possible. C'est sûr que je suis un interprète et c'est normal. Il se sert de différents interprètes, je transmets et s'il y a des tâtonnements, des erreurs, des imprécisions, cela fait partie du message, mais le message se corrige continuellement lui-même. Telle erreur, à tel moment, sera corrigée 
ensuite. II ne faut pas souffrir de tensions continuelles: à tel moment je transmets ceci, c'est parfait, c'est bien, c'est comme cela. J'ai juste à continuer à m'affiner comme instrument. Parce que c'est le haut point, jamais je n'arriverai à dire: j'ai la vérité, la voilà, Dieu vous le dit. Puisque je n'arriverai jamais à faire cela, pourquoi serais-je anxieux, coupable de faire une erreur? De toute façon dans l'ordre de la manifestation, tout ne procède que par erreur. Compenser, corriger. Cela permet une sorte de gratuité, de spontanéité dans le mouvement, de détente. Puisqu'on a confiance dans le Grand feed back.

J. D. Lorsque tu donnes un récital de poésie, sens-tu une relation érotique se développer entre toi et ton public?

P. C. Pas tout le temps, mais il s'établit une chaleur magnétique parfois et je me sens très réceptif. C'est comme si ce que je dis était recréé à ce moment-là, était retransmis. Comme si les mots étaient purement un support, un flot venant de lê langue universelle, pour utiliser l'expression de Rimbaud, qui passe à ce moment-là. Mais comme au départ je ne suis pas un personnage «chaud" (hot) cela n'atteint pas l'irtensité, l'éclatement (sauf une fois) qu'a illustré au maximum une Janis Joplin. A un moment donné je me sentais infériorisé puis j'ai graduellement constaté que si Janis Joplin était nécessaire, par contre il y avàit d'autres modes, d'autres territoires. Et que peut-être j'avais accès à des territoires plus froids, peut-être pourrais-je appeller fraîcheur ce froid-là. Le voir, non pas comme un défaut, mais comme une qualité spécifique. Et que cette fraîcheur-là est l'enveloppe d'un feu, d'une violence très grande. Le volcan dans un verre de cristal. Le volcan peut alimenter quelque chose de très spécial. En fin de compte, je peux créer une intimité fraîche, avec des gens, dans une situation publique.

Mais c'est encore très potentiel. Je pourrai aller plus loin. Cela rejoint la question de la pédérastie et, à travers elle, ce que j'appelle l'isosexualité, c'est l'image du Prince de Sexamour, une sexualité ouverte, orgiaque, (ce qui ne veut pas dire le climax seulement - elle inclut la tendresse, la caresse). Mais dans la transparence. Je me sens solaire plutôt que lunaire. Je place tout cela dans une ouverture totale, dans la franchise, abrupte, brutale.

J. D. Tu parlais de Janis Joplin. Tu cites aussi dans tes livres de nombreux chanteurs de rock, Jim Morrison, les Beatles, Crosby, Still and Nash. Ressens-tu le besoin d'élargir ton public comme Charlebois et Duguay?

P. C. J'ai commencé à prendre conscience que mon champ d'influence, mon champ d'action n'était pas aussi large que celui de Charlebois ou de Duguay. Peut-être va-t-il aller en s'élargissant? Je n'en sais rien. Pour l'instant, il est plus restreint, même s'il dépasse les limites d'un milieu spécialisé, littéraire. Parce qu'il atteint beaucoup de jeunes et des jeunes qui ne sont pas spécialement plongés dans la littérature. 
Mon médium dépasse effectivement les moules de la littérature traditionnelle.

Je pense, c'est l'image que j'en ai, souvent, que par mes livres, ou un "show", un journal, j'ai un champ d'action. Je me promène dans la société parallèle, la société «alternative". Et au Québec actuellement, je suis connu en fonction de ce réseau-là. Mes articles, dans Mainmise, m'ont permis cette espèce de développement.

C'est toujours, à chaque fois, l'individu que j'atteins. J'ai remarqué ceci que dans une situation de groupe c'est individu par individu que j'atteins les gens. Comme si j'étais là pour favoriser la différence individuelle. Et c'est ce qui se passe au niveau de l'audience. Dans les témoignages que j'ai eus, ce sont toujours des gens qui se sont alimentés de mes textes, dans un contexte chercheur de thérapie, de changement, de recherche, d'expérimentation, et finalement se réalise graduellement ce qui était dans mes intentions. Communiquer, développer la communication entre les compagnons-chercheurs.

II subsiste une certaine difficulté, un certain travail à fournir pour la compréhension de mes textes. Il ne s'agit pas vraiment de divertissement. Une chanson de Charlebois va opérer un travail, par un flash, assimilable par un grand nombre, ce qui est très bon. Cela va opérer dans une très large audience et les gens vont se mettre à changer en conséquence.

J'ai le sentiment, moi, d'arriver dans un territoire ouvert par Charlebois et d'autres. Je sais que là Charlebois est passé, puis que Duguay est passé, avec quelque chose de plus élaboré du reste, de plus complexe. Je vais passer moi-même et opérer d'autres changements au niveau du médium du poème. C'est comme si le terrain avait été travaillé, et j'entre avec plus de profondeur dans ce terrain. Je vois ceci comme une complémentarité dans le travail.

Un autre que moi arrivera, après moi, avec quelque chose d'encore plus complexe, et des allers et retours entre cela, probablement.

Je ne suis pas aussi populaire que Charlebois. Je n'ai pas de chansons dans un juke-box. Le travail se fait aussi au niveau du juke-box. Je ne vois pas de scission mais des niveaux, dans une même affaire.

Interview réalisée par Caroline Bayard, (University of Toronto) et Jack David (York University), à Toronto, le 12 mars 1976. 